\title{
Auricular acupressure for insomnia in hemodialysis patients: study protocol for a randomized controlled trial
}

Yuchi $\mathrm{Wu}^{1,2 \dagger}{ }^{2}$ Lihong Yang ${ }^{1,2 \dagger}$, Lingli $\mathrm{Li}^{2}$, Xiuqing $\mathrm{Wu}^{1,2}$, Zhicong Zhong ${ }^{1,2}$, Zhiren $\mathrm{He}^{2,4}$, Hongyan $\mathrm{Ma}^{2,4}$, Lixin Wang ${ }^{2,4}$, Zhaoyu Lu ${ }^{1,2}$, Cun Cai ${ }^{2,5}$, Daixin Zhao ${ }^{2,5}$, Xiangxin Meng ${ }^{6}$, Airong Qi ${ }^{7}$, Aicheng Yang ${ }^{8}$, Guobin Su ${ }^{1,2}$, Xinfeng Guo ${ }^{1,2,3}$, Xusheng Liu ${ }^{1,2}$, Chuan Zou ${ }^{2^{*}}$ and Qizhan Lin ${ }^{1^{*}}$

\begin{abstract}
Background: Patients on maintenance hemodialysis (MHD) frequently complain of insomnia. Poor sleep quality impairs their quality of life and adversely affects long-term outcome. Previously we applied auricular acupressure therapy (AAT) for MHD patients with insomnia and yielded favorable results. AAT probably improves sleep quality by stimulating the vagus nerve and inhibiting sympathetic overactivity. However, the efficacy of AAT for insomnia in this population is still lacking. The proposed randomized controlled trial (RCT) will evaluate the efficacy and safety of AAT for improvement of sleep quality in MHD patients with insomnia.
\end{abstract}

Methods/design: The proposed study is a multi-center, double-blind (participants and assessors), parallel-group RCT. A total of 112 participants with insomnia will be recruited from six hemodialysis centers in Guangzhou, China, and randomly allocated in a 1:1 ratio to receive auricular acupressure on either active points (AA group) or control points (points irrelevant to insomnia management, SAA group). The treatment will last for 8 weeks prior to a followup period of 12 weeks. Evaluation by blinded assessors at baseline, at 8 weeks (end of treatment) as well as at 4-week, 8week and 12-week follow-ups (after intervention) will include Pittsburgh Sleep Quality Index (PSQI) scores and average weekly dose of hypnotics. The primary endpoint is clinical response rate (percentage of participants who reach a reduction of PSQI global score $\geq 3$ in each group) at 8 weeks from baseline. Secondary endpoints include the changes in PSQI scores over time from baseline, as well as the changes in weekly dose of hypnotics.

Discussion: This paper describes the rationale and design of a double-blind RCT that aims to determine the efficacy and safety of AAT for insomnia of hemodialysis patients. If successful, this project will provide evidence of the efficacy and safety of AAT for insomnia of hemodialysis patients.

Trial registration: ClinicalTrials.gov, Identifier: NCT03015766. Registered on 22 December 2016.

Keywords: Insomnia, Hemodialysis, Auricular acupressure, Randomized controlled trial

\footnotetext{
*Correspondence: doctorzc541888@126.com; linqizhan656635@163.com

${ }^{\dagger}$ Equal contributors

${ }^{2}$ Second Clinical Medical College, Guangzhou University of Chinese Medicine,

Guangzhou, People's Republic of China

${ }^{1}$ Hemodialysis Department, Guangdong Provincial Hospital of Chinese

Medicine, Guangzhou, People's Republic of China

Full list of author information is available at the end of the article
}

(c) The Author(s). 2018 Open Access This article is distributed under the terms of the Creative Commons Attribution 4.0 International License (http://creativecommons.org/licenses/by/4.0/), which permits unrestricted use, distribution, and reproduction in any medium, provided you give appropriate credit to the original author(s) and the source, provide a link to the Creative Commons license, and indicate if changes were made. The Creative Commons Public Domain Dedication waiver (http://creativecommons.org/publicdomain/zero/1.0/) applies to the data made available in this article, unless otherwise stated. 


\section{Background}

Sleep disorders, such as insomnia, restless leg syndrome, sleep apnea and excessive daytime sleepiness, are common in patients with end-stage renal disease (ESRD), especially in those receiving dialysis [1]. Insomnia is one of the most common conditions, with a prevalence rate as high as 49$84.5 \%$ depending on the specific population [2-5]. Poor sleep quality is known to have adverse effects on patients' health-related quality of life [6], mental health [7], social adaptability [8] and even long-term survival [9].

ESRD patients with insomnia share common risk factors identified in the general population, such as female sex, advanced age, lack of physical activity and depressed mood [10]. In addition, multiple factors, including chronic kidney disease (CKD) and renal replacement therapy, also play roles in the development of insomnia. CKD comorbidity (e.g., diabetes) and CKD-related complications (e.g., uremia, anemia and hyperparathyroidism) are all recognized factors $[5,11,12]$. Somatic symptoms, such as pruritus, bone pain and arthralgia, are also related to impaired sleep quality in dialysis patients [13].

Poor sleep quality prompts physicians to prescribe hypnotics (usually benzodiazepines) for hemodialysis (HD) patients, which may be associated with adverse effects, such as memory impairment, drug resistance, dependence and addiction [14]. Current guideline for chronic insomnia suggests that sleep medications should be properly used based on awareness of both effectiveness and harms [15]. Despite concerns regarding safety and dependency of long-term usage of hypnotic drugs expressed by both physicians and patients, a considerable proportion (benzodiazepines: $42.6 \%$ and hypnotics: $20.0 \%$, in single-center cross-sectional study [16]) of HD patients continue to take nightly sleep medications for prolonged periods while at the same time complaining of unsatisfactory sleep quality. Therefore, both caregivers and patients are confronted with the conflicting requirements of improving patients' sleep quality and reducing their dependence on hypnotics.

Non-pharmacological therapies with supportive evidence of a hypnotic effect are desirable. Auricular acupressure therapy (AAT), a therapeutic approach that treats various disorders by stimulating specific points on the ear, is a popular form of complementary medicine in China. Kung's study in Taiwan among women with postmenopausal insomnia indicated that AAT led to increased cardiac parasympathetic activity and reduced cardiac sympathetic activity with improvement of sleep quality [17]. In another investigation in women with postpartum insomnia [18], patients receiving AAT for 2 weeks showed decreases in Pittsburgh Sleep Quality Index (PSQI) total scores from 8.7 (pre test) to 5.57 (post test, $36 \%$ reduction). Scores on the subscales of PSQI also showed significant improvement of sleep quality. Autonomic nervous system regulation by the auriculovagal afferent pathway [19] has been proposed to explain how AAT improves sleep quality. The auricular branch of the vagus nerve links the acupressure stimulation and vagal regulation. AAT might trigger vagal regulation and correct the overactivity of sympathetic nerves which plays a crucial role in chronic hemodialysis patients with insomnia [20]. Therefore, auricular acupressure might be effective in improving sleep quality for hemodialysis patients.

Our previous study showed improvements in sleep quality and reduced consumption of sleep medications $(6.98 \pm 4.44$ pills per week post AAT compared with $4.23 \pm 2.66$ pills per week pre AAT, $P<0.01$ ) after a 4-week course of AAT in addition to basic care in hemodialysis patients with severe insomnia [21]. In a pilot randomized controlled trial (RCT), we found that AAT on specific points with an appropriate rationale yielded a higher response rate in PSQI score improvement than that on sham points $\left(62.5 \%\right.$ vs. $32.3 \%, X^{2}=$ $5.77, P=0.02$ ) [22]. However, these previous studies had some limitations. Data collected from a single center may lack the scientific rigor or external validity required to support widespread application of AAT. In addition, sample size was not calculated to guarantee sufficient statistical power. Owing to design limitations, the efficacy and safety of AAT in hemodialysis populations with insomnia have yet to be confirmed. Therefore, we aim to perform a full-scale RCT to test the hypothesis that AAT is an effective and safe means of improving sleep quality and reducing dependence on hypnotics in hemodialysis patients.

\section{Methods/design \\ Study design}

The study uses a two-arm, randomized, double-blind, sham-controlled design. The trial will be performed in six hemodialysis centers in Guangdong Province, People's Republic of China, all of which are tertiary hospitals of Chinese Medicine: Guangdong Provincial Hospital of Chinese Medicine, Guangzhou Charity Hospital, Guangzhou HEMC (Higher Education Mega Center) Hospital, Guangzhou Hospital of Traditional Chinese Medicine, Wuyi Hospital of Traditional Chinese Medicine and Shenzhen Hospital of Traditional Chinese Medicine. This trial will include an 8-week treatment period and a 3-month follow-up period (visits at 4-week intervals). Eligible subjects will be randomized into one of the two arms in a ratio of 1:1 to receive AAT on either active points or sham points. The trial has been registered at ClinicalTrials.gov (NCT03015766).

\section{Participants}

We will include patients receiving regular hemodialysis treatment for more than 3 months and less than 10 years. These patients are more likely to go through the whole trial which will continue for 5 months. Incident 
hemodialysis patients (less than 3 months) and those who have been on dialysis for more than 10 years usually have a high mortality rate $[23,24]$. Patients will have initial assessment of sleep quality and physical examination to meet the diagnosis of chronic insomnia. Potentially eligible participants are being identified through screening of patient medical records at each dialysis center. Screening of patients in each center will be performed by one assigned personnel according to the inclusion and exclusion criteria. A flow chart of the participant recruitment is shown in Fig. 1.

\section{Participant timeline}

The time course for participant recruitment, intervention, assessment and follow-up is shown in the Standard
Protocol Items: Recommendations for Interventional Trials (SPIRIT) Figure (Fig. 2).

\section{Inclusion criteria}

Subjects fulfilling all of the following criteria will be included:

1. Aged $18-75$ years (either sex).

2. On regular hemodialysis (two or three sessions every week, $4 \mathrm{~h}$ each session, total weekly dialysis period $\geq$ $10 \mathrm{~h}$ )

3. Insomnia diagnosed according to The Diagnostic and Statistical Manual of Mental Disorders, Fifth Edition (DSM-5) [25]

4. Baseline global PSQI score $>7$

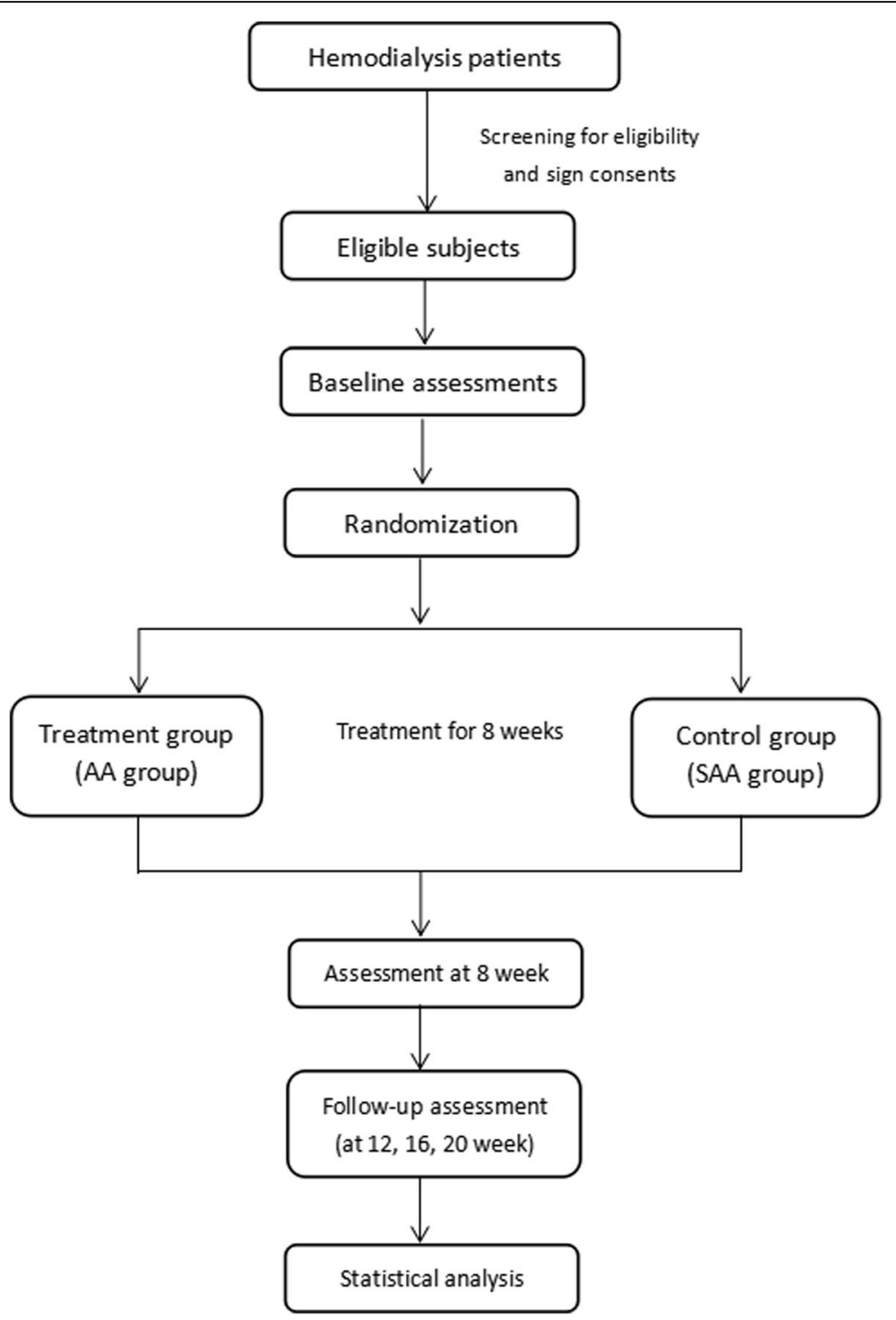

Fig. 1 Trial flow chart 


\begin{tabular}{|c|c|c|c|c|c|c|}
\hline \multirow{2}{*}{ Research stages } & \multirow{2}{*}{$\begin{array}{l}\text { Baseline } \\
\text { (Day 0) }\end{array}$} & \multicolumn{2}{|c|}{ Treatment period } & \multicolumn{3}{|c|}{ Follow-up period } \\
\hline & & Day $l \pm 7 d$ & 8 weeks $\pm 7 d$ & 12 weeks $\pm 7 \mathrm{~d}$ & 16 weeks $\pm 7 d$ & 20 weeks $\pm 7 d$ \\
\hline \multicolumn{7}{|l|}{ Patients } \\
\hline Informed consent & $x$ & & & & & \\
\hline $\begin{array}{l}\text { Sign informed } \\
\text { consent }\end{array}$ & & $x$ & & & & \\
\hline Medical history & $x$ & & & & & \\
\hline \multicolumn{7}{|l|}{$\begin{array}{l}\text { Physical } \\
\text { examination }\end{array}$} \\
\hline Randomization & & $x$ & & & & \\
\hline \multicolumn{7}{|l|}{ Intervention } \\
\hline AA group & & \multicolumn{2}{|c|}{$\begin{array}{l}\text { Successive AA treatment on } \\
\text { TF4, AH6a, CO15, AT4 }\end{array}$} & & & \\
\hline \multicolumn{7}{|l|}{ Comparison } \\
\hline SAA group & & \multicolumn{2}{|c|}{$\begin{array}{l}\text { Successive AA treatment on } \\
\text { HX 5-9. }\end{array}$} & & & \\
\hline \multicolumn{7}{|l|}{\begin{tabular}{|l} 
Outcomes \\
\end{tabular}} \\
\hline $\begin{array}{l}\text { Clinical response } \\
\text { rate }\end{array}$ & & & $x$ & & & \\
\hline PSQI scores & & $x$ & $x$ & $x$ & $x$ & $x$ \\
\hline $\begin{array}{l}\text { Usage of sleep } \\
\text { medicine }\end{array}$ & & $x$ & $x$ & $x$ & $x$ & $x$ \\
\hline \multicolumn{7}{|l|}{ Participant safety } \\
\hline Adverse events & & & $x$ & $x$ & $x$ & $x$ \\
\hline
\end{tabular}

Fig. 2 Standard Protocol Items: Recommendations for Interventional Trials (SPIRIT) Figure. Timing of visits and data collection. Abbreviations: AA auricular acupressure, SAA sham auricular acupressure, PSQI Pittsburgh Sleep Quality Index

\section{Informed consent provided}

\section{Exclusion criteria}

Patients with any of the following conditions will be excluded:

1. Presence of comorbidities including cancer, congestive heart failure, connective tissue disease and hematological diseases

2. Inadequately dialyzed, indicated by urea clearance index $(\mathrm{KT} / \mathrm{V})<1.20$

3. Presence of severe physical symptoms, such as bone pain, itchy skin, sleep apnea and restless legs, which are obviously causative of insomnia; and exhaustion caused by severe anemia (hemoglobin $<60 \mathrm{~g} / \mathrm{L}$ ) or malnutrition (serum albumin $<30 \mathrm{~g} / \mathrm{L}$ )

\section{Randomization and allocation concealment}

Subjects will be randomly allocated into one of the two treatment arms in a 1:1 ratio: (1) treatment group (AA group) and (2) control group (SAA group). Central randomized allocation will be performed. Patients will be stratified based on six sites and randomly assigned to one of the two groups. The Chinese Clinical Trial Registry will generate the random number sequence using computer software, and will deposit the allocation sequence in ResMan Clinical Trial Management Public Platform, a web-based database. When a new participant is enrolled in the study, the research coordinator of each site will apply for the allocation information through this online system and assign participants to interventions accordingly. It is not possible to speculate on the allocation status of the participant before obtaining this information. The other personnel, including research nurses, assessors and clinical physicians, are not authorized to apply for random numbers. All processes will be recorded and saved appropriately.

\section{Blinding}

Both the participants and the assessors in this trial will be blinded to the allocation. AAT will be provided by trained nurses and manipulated by patients. The nurses and patients will be told that we are comparing two different treatment protocols (TPs), in which all auricular points involved are named as numbers with specific 
defined locations. We will have two nurses in each site assigned to perform the intervention. One will be responsible for providing TP A and the other for TP $\mathrm{B}$. The assignment of nurses is done by drawing lots. The nurses will receive researcher training before the trial. They are required to perform auricular acupressure following the standard procedure, no matter what TP they are practicing. They are also required not to discuss the difference between the two TPs with patients. To avoid differences due to the Hawthorne and Rosenthal effect $[26,27]$, we will script the nurses to limit their interaction with the participants. Any additional questions will be directed to the research coordinator of each center. All results will be reported to another investigator responsible for assessing sleep quality in a clinical interview. The investigator will provide questionnaires to participants at the scheduled time for assessment. Clinical physicians of the Hemodialysis Department are allowed to prescribe estazolam (14 tablets each time) when patients require. At the end of the week, the patients should return the tablets left and obtain a new prescription if they need. All personnel will be properly assigned before the start of the trial.

\section{Interventions}

AAT is based on the theory that specific points on the auricle correspond to major organs or systems of the body and that the function of the targeted organ or system can be modulated by manipulating corresponding auricular acupoints. AAT applies stimulation through pressure on specific acupoints using embedded beads, usually Semen vaccariae seeds (Wang Bu Liu Xing) or stainless steel beads. This approach is non-invasive, and recipients can be trained to manipulate the beads themselves to exert stimulation at a required frequency. A map of commonly used auricular acupoints is shown in Fig. 3a.

In this trial, two research nurses for each center will be assigned to administer AAT to participants. They will gain approval from licensed acupuncturists through training and competence assessment before the start of the trial. They will provide AAT for patients during dialysis sessions for eight consecutive weeks. A standard procedure of AAT has been established as follows:
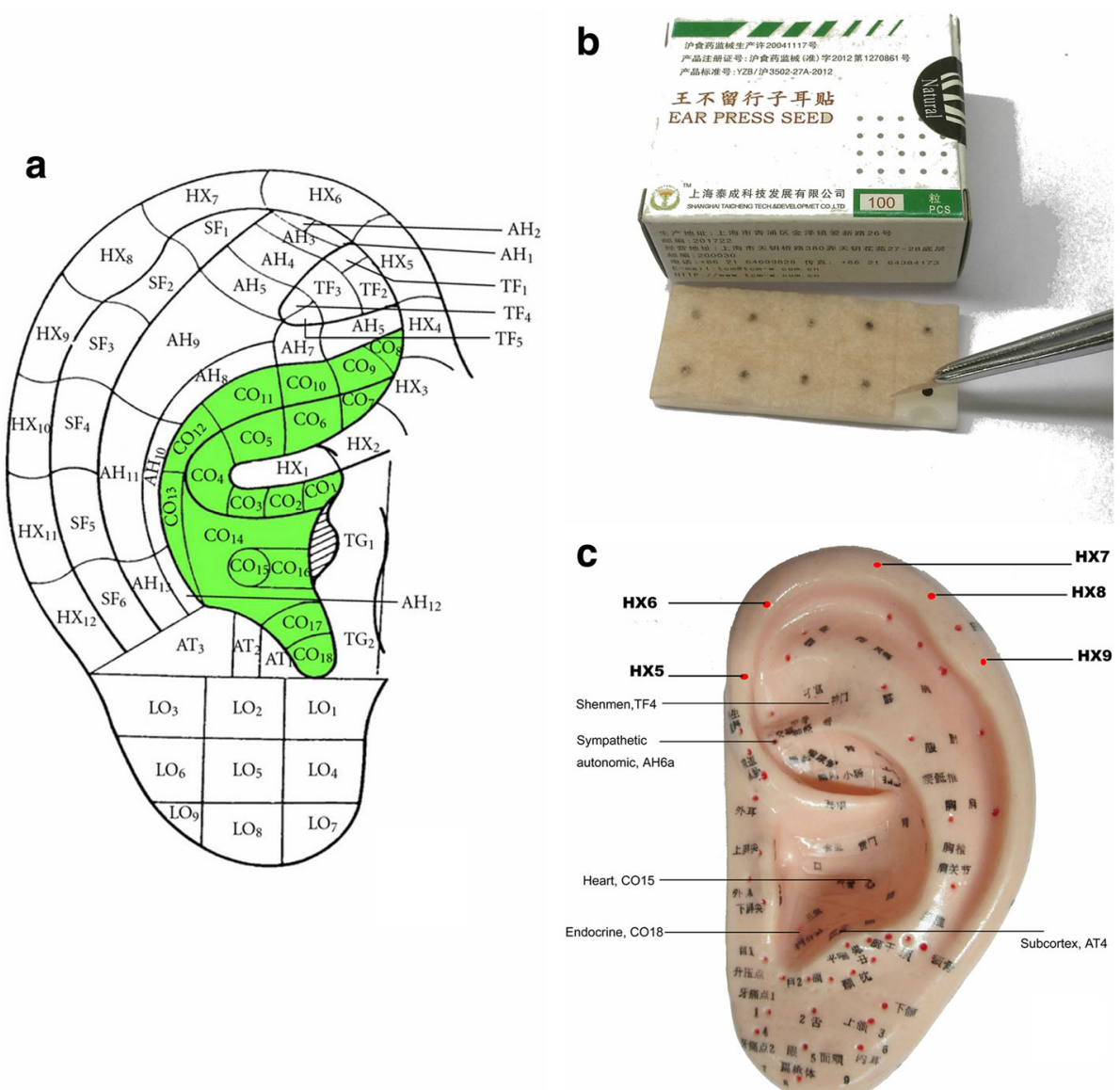

Fig. 3 A demonstration of interventions in the trial. a A map of auricular acupoints commonly used. b Ear-press seed (Wang Bu Liu Xing) applied in this trial. c Acupoints of both groups in the trial 
1. Identify the ear points according to the TP directed by the research coordinator.

1. Sterilize the involved area of the ear with $75 \%$ isopropyl alcohol

2. Attach an adhesive plaster (size: $1.0 \mathrm{~cm} \times 1.0 \mathrm{~cm}$ ) with one bead (Semen vaccariae, globes approximately $2.0 \mathrm{~mm}$ in diameter; surface: smooth; colour: black; Taicheng Technology \& Development Co., Ltd., Shanghai, China) embedded in each acupoint (Fig. 3b)

3. Provide instructions to press the beads with appropriate strength (approximately $0.3-0.4 \mathrm{~kg}$ ) and rate (one to two beats per second) for $1 \mathrm{~min}$ at each point [28]. This should be done three times during the day and once in the evening

4. The plasters will be replaced with fresh ones every 2-3 days (usually on the dialysis day) depending on the dialysis schedule. If the plasters or beads become detached, participants should come to the hospital to receive fresh plasters. To improve their adherence to interventions, we plan to attach the auricular acupressure beads when the patients come for their scheduled dialysis and replace the beads every 2-3 days (on the dialysis day as well). Activities during each visit in the treatment period will be documented in the Case Report Form.

\section{Treatment group}

Participants in the treatment group (AA group) will receive AAT on five active acupoints:

Acup.1. Shen Men (Spiritual Gate, TF4)

Acup.2. Jiao Gan (Sympathetic autonomic, AH6a)

Acup.3. Xin (Heart, CO15)

Acup.4. Pi Zhi Xia (Subcortex, AT4)

Acup.5. Nei Fen Mi (Endocrine, CO18)

The rationale for selecting these points has been explicitly described previously [21, 22]. Briefly, these points were selected based on Traditional Chinese Medicine (TCM) meridian theory, results of an academic literature review, and on-site clinical practice experience [29]. These points help patients sleep by regulating the autonomic nervous system. These points are anatomically connected to the auricular branch of the vagus nerve. Stimulation on these points may increase parasympathetic activities and reduce sympathetic activities $[30,31]$, which play an important role in sleep [32].

\section{Control group}

Participants in the control group (SAA group) will receive AAT on five helix points (HX 5-9), which are clearly remote from the inner ear area. These points lack evidence for insomnia management. In our pilot study, these points had minor effects on sleep quality, which could not be differentiated from the placebo effect.

The acupoints in both groups are illustrated in Fig. 3c. The locations of these points in both groups are listed in Table 1, following the National Standards of the Nomenclature and Location of Auricular Acupoints published in China [33].

\section{Sample size estimation}

In a pilot RCT conducted previously, the clinical response rates of AAT and sham AAT were $62.5 \%$ and $32.3 \%$, respectively [22]. With these data, we used the following formula to calculate the estimated sample size [34].

$$
\mathrm{N}=\frac{\left(u_{\alpha}+u_{\beta}\right)^{2}(1+1 / \mathrm{k}) p(1-p)}{\left(p_{e}-p_{c}\right)^{2}}, p=\frac{p_{e}+k p_{c}}{1+k}
$$

In this formula, $k$ (the ratio of allocation) $=1, u_{\mathrm{a}}$ and $u_{\beta}$ are 1.6449 and 1.2816 , respectively, when $90 \%$ statistical power $(\beta=0.1)$ is required and a significance level of $5 \%(\alpha=0.05)$ is allowed. Here, $p_{\mathrm{e}}$ represents the incidence rate of the treatment group, while $p_{\mathrm{c}}$ represents that of the control group. Therefore, we will require at least 56 subjects in each group taking into account an allowed dropout rate of $15 \%$. The results have been confirmed using PASS software 11.0 (NCSS, LLC, Kaysville, UT, USA) by tests for two independent proportions.

Table 1 Locations of auricular acupoints used in the trial

\begin{tabular}{|c|c|}
\hline $\begin{array}{l}\text { Auricular } \\
\text { acupoints }\end{array}$ & Locations \\
\hline \multicolumn{2}{|l|}{ AA group } \\
\hline TF4 & $\begin{array}{l}\text { At the apex of the triangular fossa, in the bifurcating } \\
\text { point between superior and inferior crura of the } \\
\text { antihelix }\end{array}$ \\
\hline $\mathrm{AH} 6 \mathrm{a}$ & At the end of the inferior antihelix crus \\
\hline $\mathrm{CO} 15$ & Around the central depression of the cavum conchae \\
\hline AT4 & At the medial side of the antitragus \\
\hline $\mathrm{CO} 18$ & $\begin{array}{l}\text { In the intertragic notch, at the medial inferior part of } \\
\text { the cavum conchae }\end{array}$ \\
\hline \multicolumn{2}{|l|}{ SAA group } \\
\hline $\mathrm{HX} 5$ & $\begin{array}{l}\text { An area on the helix, between the two feet of the } \\
\text { antihelix }\end{array}$ \\
\hline$H X 6$ & $\begin{array}{l}\text { An area on the helix, between the upper foot of the } \\
\text { antihelix and the apex conchae auris }\end{array}$ \\
\hline$H X 7$ & $\begin{array}{l}\text { An area on the helix, between the apex conchae auris } \\
\text { and the helix tubercle }\end{array}$ \\
\hline$H X 8$ & An area on the helix, and at the helix tubercle \\
\hline HX 9 & An area on the helix, and below the helix tubercle \\
\hline
\end{tabular}

$A A$ auricular acupressure, $A A T$ auricular acupressure therapy 


\section{Outcome measurement}

Sleep quality will be measured by PSQI score [35]. PSQI contains seven domains with scores from 0 to 3 , yielding a total score ranging from 0 to 21 , where a higher score indicates poorer sleep quality. The reliability and validity of its Chinese version have been confirmed [36, 37]. PSQI will be assessed at baseline, immediately after treatment (i.e., 8 weeks from baseline) and at 4, 8 and 12 weeks after treatment.

\section{Primary outcome measurement}

The primary outcome will be the difference of the clinical response rates at 8 weeks from baseline between two groups. According to the academic literature review, response is defined as a reduction of PSQI global score $\geq 3$ [38]. The clinical response rate will be the number of participants who achieve a reduction of PSQI global score exceeding 3 points divided by the total number of participants in each group and multiplied by $100 \%$.

\section{Secondary outcome measurement}

Secondary outcomes will be changes in PSQI scores (including global score and scores of each of the seven domains) at the end of treatment and at each follow-up visit compared with baseline.

As the trial will not exclude participants on hypnotics or plan a washout period due to ethics considerations, participants may maintain their dose of hypnotics or require hypnotic agents during the study. In this case, clinical physicians will prescribe estazolam (14 tablets each prescription, $1 \mathrm{mg}$ each tablet) and instruct them to adjust the dose of hypnotics according to their sleep quality. The participants should return the unconsumed tablets at the end of the week or require another prescription as needed. The weekly dose of hypnotic agents will be reported and also serve as a secondary outcome.

\section{Patient safety}

All participants will undergo blood biochemical tests before randomization and at the end of follow-up. According to Blood Purification Standard Operating Procedure in China [39], maintenance hemodialysis (MHD) patients will undergo investigations including serum creatinine $(\mathrm{SCr})$, blood urea nitrogen $(\mathrm{BUN})$, total carbon dioxide $\left(\mathrm{TCO}_{2}\right)$, potassium, calcium, phosphate, parathyroid hormone (PTH), albumin, hemoglobin and KT/V every 3 months. These parameters are all essential for monitoring the complications and adequacy of dialysis for MHD patients. We will utilize these data to assess their safety during the trial. Adverse events throughout the treatment and follow-up periods, regardless of relevance to the interventions, will be documented, reported to the Ethics Committee and dealt with using appropriate treatment.

\section{Withdrawal criteria}

Participants will be withdrawn from the study in the following situations:

1. When a participant asks to withdraw from the study, at any time, for any reason

2. When severe adverse events or reactions occur

The data of these participants will be collected and included in further analysis.

\section{Data collection and management}

Data of demographic characteristics and baseline assessment will be collected by screeners when the patients are recruited. Clinical outcome measurement will be performed by assigned outcome assessors after the treatment is completed and during the follow-up period. Assessors of each center will undergo a training course to obtain the capability of using PSQI. Data regarding medicine prescription and any adverse events reported by patients will be collected by clinical physicians of the Hemodialysis Department.

A research coordinator of each center will conduct quality control of data collection and be responsible for data entry. The data manager will be responsible for initial data cleaning, identifying, coding and conversion into the proper format for data analysis. Data analysis will be performed by a biostatistician from the Evidencebased Medicine and Clinical Research Service Group.

\section{Statistical analysis}

Statistical analysis will be performed using SPSS Statistics Software 22.0 (IBM Corp., Armonk, NY, USA). For the results of the study, means \pm standard deviations will be calculated for normally distributed quantitative variables and median (interquartile range) for non-normally distributed variables. In addition, the 95\% confidence interval around the mean will be presented. For categorical variables, the number and percentage of patients within each category will be presented.

Statistical analyses of primary and secondary outcomes will be conducted as follows. First, all analyses will be based on the intention-to-treat (ITT) principle. All patients enrolled in the study will be included in the analyses, regardless of whether they complete the treatment or adhere to the protocol. Data distribution is expected to be normal, and skewed distribution data will be transformed prior to analysis. Then, the outcome of the clinical response will be analyzed by the chi-squared test or Fisher's exact test. For the secondary outcomes, repeated-measures analyses of variance (rANOVA) will be conducted to compare the changes in PSQI scores and the weekly dose of hypnotics at 8, 12, 16 and 20 weeks from baseline. Missing values will be addressed 
using the method of last observation carried forward (LOCF). To avoid potential confounding factors, additional sensitivity analyses will be performed following these statistical analyses.

\section{Discussion}

Impaired sleep quality of patients on dialysis adversely impacts their quality of life and long-term survival. Although AAT has been practiced as a complementary treatment for insomnia, there is still an important gap with regard to sufficient evidence. The results of this trial are expected to provide convincing evidence regarding whether AAT is effective for MHD patients with insomnia.

This study has been designed carefully following the Consolidated Standards of Reporting Trials (CONSORT) Statement of RCTs and reported according to the Standard Protocol Items: Recommendations for Interventional Trials (SPIRIT) Statement (Additional file 1). One of the major issues in the trial design is the setting of the control group. Studies on the efficacy of acupuncture have been controversial based mainly on the following two points: first, the psychological effect (placebo effect, from both the investigators and the patients) could make a considerable contribution to the results. Second, the therapeutic effect may not depend on specific points. Our previous pilot study showed that points on the helix, which are irrelevant to the physiology of sleep, are appropriate for the sham intervention. In addition, we specified the points in both groups as numbers and letters. We also scripted the interactions between practitioners/assessors and subjects. These approaches help to blind both the participants and the practitioners, reducing their psychological interference to the minimum. Therefore, it will be easier to interpret the contributions of specific acupoints to the outcome.

Another concern is whether hypnotics should be washed out and prohibited during the trial. Indeed, this approach is good for trial design but impractical for carrying out the trial, because it increases the suffering of patients and reduces their compliance. As the trial is intended to assess whether AAT can help improve sleep quality and reduce drug use, rather than whether AAT is better than hypnotics, we decided to allow the use of hypnotics under conditions of strict surveillance.

For outcome measurement, PSQI is a well-validated, patient-reported questionnaire. But for now there is not a defined minimal clinically important difference (MICD) to reflect the changes in a clinical intervention that are meaningful for the patients. In some case, statistically significant difference has little clinical significance. Therefore, we adopted the response rate for the primary endpoint from a clinical trial published by Prof. Buysse, the author of the PSQI. He performed a comprehensive data review to set the criteria for response: participant with a change in PSQI of more than 3 points after treatment was defined as a response case [38]. In addition, the global PSQI score and scores of seven domains will be also analyzed as secondary outcomes in this trial.

The design of the trial minimizes the risk of bias by the following methods: (1) It is designed as a multi-center, double-blinded RCT. A central randomization method is applied to ensure completely random allocation and concealment. Both the patients and the assessors are blinded to the groups, (2) The sample size was estimated based on a previous pilot study [22], (3) The screening of participants is performed strictly to represent the population studied, (4) The manipulation of AAT is standardized and the practice of AAT is performed strictly under the guidance of authoritative clinical guidelines [40, 41]. A senior acupuncturist will be invited to train the research nurses from six sites and assess their competence, which will ensure the homogeneity of treatment and (5) The selected scale for outcome measurement is of good validity and reliability [42]. The investigators involved in the study will undergo a training course and have their role assigned clearly before the start of the trial.

There are several limitations of this study. First, sleep quality is not assessed by objective measurement such as polysomnography or actigraphy. There are some difficulties for us to perform these tests. Most hemodialysis patients refused to go through polysomnography in our pilot studies when they were required to stay a whole night in hospital. Actigraphy seems easily accepted but this equipment is unavailable in all centers. Due to limited financial support, we have to make the best of a well-acknowledged scale to assess the outcome. In a future study, actigraphy should be included. Second, anxiety and depression are commonly accompanied with sleep disorders but are not assessed in this study. Recent study has suggested that they have positive correlations with PSQI score [43]. A more comprehensive assessment of the effect of auricular acupressure will include sleep quality, anxiety and depression, and quality of life in future study.

This protocol offers a standardized process to guide subsequent clinical research. The results of this trial will generate concrete evidence regarding the efficacy of AAT in insomnia management of dialysis patients.

\section{Trial status \\ Ongoing recruitment}

\section{Additional files}

Additional file 1: SPIRIT Checklist. SPIRIT 2013 Checklist: recommended items to address in a clinical trial protocol and related documents. (DOC $97 \mathrm{~kb}$ ) 
Additional file 2: Complementary information for the trial. (DOC $35 \mathrm{~kb}$ )

Additional file 3: WHO Trial Registration Data Set. (PDF 79 kb)

Additional file 4: Informed consent materials. (DOC $30 \mathrm{~kb}$ )

\section{Abbreviations}

AA: Auricular acupressure; AAT: Auricular acupressure therapy; BUN: Blood urea nitrogen; CKD: Chronic kidney disease; CONSORT: Consolidated Standards of Reporting Trials; DSM: Diagnostic and Statistical Manual of Mental Disorders; ESRD: End-stage renal disease; HD: Hemodialysis; ITT: Intention-to-treat; LOCF: Last observation carried forward; MHD: Maintenance hemodialysis; PSQI: Pittsburgh Sleep Quality Index; PTH: Parathyroid hormone; RCT: Randomized controlled trial; SAA: Sham auricular acupressure; SCr: Serum creatinine; TCM: Traditional Chinese Medicine; $\mathrm{TCO}_{2}$ : Total carbon dioxide; TP: Treatment protocol

\section{Acknowledgements}

QZL provided general support as the head of the Hemodialysis Department in Guangdong Provincial Hospital of Chinese Medicine. He was responsible for the design of this RCT and for communicating important protocol modifications to relevant parties. YCW and LHY both contributed equally to this work and should be considered as co-first authors. CZ and QZL are both corresponding authors.

\section{Funding}

This study is part of TCM Research Project of Guangdong Provincial Hospital of Chinese Medicine (YN2015MS25), and supported by MOST/SATCM of the People's Republic of China grant 2013BAI02B04 and the State Administration of Traditional Chinese Medicine, P.R. China (No. 201007005). The sponsor will not have a role in the design, conduct or interpretation of the study, or in any decision to submit the manuscript for publication.

\section{Availability of data and materials}

All data are fully available without restriction. The results of the trial will be published in a peer-reviewed clinical journal to ensure widespread dissemination. Individual Participant Data (IPD) will be available at ResMan ${ }^{\circledR}$ Clinical Trial Management Public Platform. The link is http://www.medresman.org/ pub/cn/proj/listbyproj.aspx?proj=2317. More details of performing this trial are included in Additional files 2 and 3.

\section{Authors' contributions}

CZ, XQW, QZL and XSL contributed to the conception and design of the study. YCW and LHY drafted and critically revised the manuscript for important intellectual content. YCW and ZRH completed the clinical trial registration and designed the Case Report Form. LLL and ZCZ prepared the tables and figures for the protocol. GBS, LHY and XFG performed the statistics plan and sample size estimation. ZYL, HYM and CC prepared materials for ethics review. LXW, DXZ, XXM, ARQ and ACY helped with implementation. All authors discussed, read, revised the manuscript and gave final approval for publication.

\section{Ethics approval and consent to participate}

The study protocol has been approved by the Ethics Committee of Guangdong Provincial Hospital of Chinese Medicine (No. B2016-137-01, date: 10 September 2016). This trial will be performed according to Ethical Principles of World Medical Association Declaration of Helsinki (Version Fortaleza 2013). All subjects will receive appropriate treatment without exacerbation of their suffering. Written informed consent will be obtained from all participants (Additional file 4). The patients will be given adequate time to raise questions and to consider whether to participate in the study.

\section{Consent for publication}

All authors read and approved the final manuscript.

\section{Competing interests}

The authors declare that they have no competing interests.

\section{Publisher's Note}

Springer Nature remains neutral with regard to jurisdictional claims in published maps and institutional affiliations.

\section{Author details}

'Hemodialysis Department, Guangdong Provincial Hospital of Chinese Medicine, Guangzhou, People's Republic of China. ${ }^{2}$ Second Clinical Medical College, Guangzhou University of Chinese Medicine, Guangzhou, People's Republic of China. ${ }^{3}$ Evidence-based Medicine and Clinical Research Service Group, Guangdong Provincial Academy of Chinese Medical Sciences, Guangzhou, People's Republic of China. ${ }^{4}$ Hemodialysis Department, Guangzhou Charity Hospital, Guangzhou, People's Republic of China. ${ }^{5}$ Hemodialysis Department, Guangzhou HEMC (Higher Education Mega Center) Hospital, Guangzhou, People's Republic of China. ${ }^{6}$ Hemodialysis Department, Guangzhou Hospital of Traditional Chinese Medicine, Guangzhou, People's Republic of China. 'Hemodialysis Department, Shenzhen Hospital of Traditional Chinese Medicine, Shenzhen, People's Republic of China. ${ }^{8}$ Hemodialysis Department, Wuyi Hospital of Traditional Chinese Medicine, Jiangmen, People's Republic of China.

Received: 18 September 2017 Accepted: 14 February 2018

Published online: 07 March 2018

\section{References}

1. Anand S, Johansen KL, Grimes B, Kaysen GA, Dalrymple LS, Kutner NG, Chertow GM. Physical activity and self-reported symptoms of insomnia, restless legs syndrome, and depression: the comprehensive dialysis study. Hemodial Int. 2013;17:50-8.

2. Losso RL, Minhoto GR, Riella MC. Sleep disorders in patients with end-stage renal disease undergoing dialysis: comparison between hemodialysis, continuous ambulatory peritoneal dialysis and automated peritoneal dialysis. Int Urol Nephrol. 2015;47:369-75.

3. Ezzat $H$, Mohab A. Prevalence of sleep disorders among ESRD patients. Ren Fail. 2015;37:1013-9.

4. Elder SJ, Pisoni RL, Akizawa T, Fissell R, Andreucci VE, Fukuhara S, Kurokawa K, Rayner HC, Furniss AL, Port FK, Saran R. Sleep quality predicts quality of life and mortality risk in haemodialysis patients: results from the Dialysis Outcomes and Practice Patterns Study (DOPPS). Nephrol Dial Transplant. 2008;23:998-1004.

5. Cengić B, Resić H, Spasovski G, Avdić E, Alajbegović A. Quality of sleep in patients undergoing hemodialysis. Int Urol Nephrol. 2012;44:557-67.

6. Ishak WW, Bagot K, Thomas S, Magakian N, Bedwani D, Larson D, Brownstein A, Zaky C. Quality of life in patients suffering from insomnia. Innov Clin Neurosci. 2012:9:13-926.

7. Blank M, Zhang J, Lamers F, Taylor AD, Hickie IB, Merikangas KR. Health correlates of insomnia symptoms and comorbid mental disorders in a nationally representative sample of US adolescents. Sleep. 2015;38:197-204.

8. Raffray T, Bond TL, Pelissolo A. Correlates of insomnia in patients with social phobia: role of depression and anxiety. Psychiatry Res. 2011;189:315-7.

9. Li Y, Zhang X, Winkelman JW, Redline S, Hu FB, Stampfer M, Ma J, Gao X. Association between insomnia symptoms and mortality: a prospective study of U.S. men. Circulation. 2014;129:737-46.

10. Mai E, Buysse DJ. Insomnia: prevalence, impact, pathogenesis, differential diagnosis, and evaluation. Sleep Med Clin. 2008;3:167-74

11. Parvan K, Lakdizaji S, Roshangar F, Mostofi M. Quality of sleep and its relationship to quality of life in hemodialysis patients. J Caring Sci. 2013;2:295-304.

12. De Santo RM, Esposito MG, Cesare CM, Cice G, Perna A, Violetti E, Conso G, Bilancio G, Celsi S, Cirillo M, Livrea A. High prevalence of sleep disorders in hemodialyzed patients requiring parathyroidectomy. J Ren Nutr. 2008;18:52-5.

13. Unruh ML, Cohen LM. Sleep and pain management are key components of patient care in ESRD. Nephrol Dial Transplant. 2012;27:2618-20.

14. Uzun S, Kozumplik O, Jakovljević M, Sedić B. Side effects of treatment with benzodiazepines. Psychiatr Danub. 2010;22:90-3.

15. Sateia MJ, Buysse DJ, Krystal AD, Neubauer DN, Heald JL. Clinical Practice Guideline for the Pharmacologic Treatment of Chronic Insomnia in Adults: An American Academy of Sleep Medicine Clinical Practice Guideline. J Clin Sleep Med. 2017;13:307-49.

16. Yeh CY, Chen CK, Hsu HJ, Wu IW, Sun CY, Chou CC, Lee CC, Wang LJ. Prescription of psychotropic drugs in patients with chronic renal failure on hemodialysis. Ren Fail. 2014;36:1545-9.

17. Kung YY, Yang CC, Chiu JH, Kuo TB. The relationship of subjective sleep quality and cardiac autonomic nervous system in postmenopausal women with insomnia under auricular acupressure. Menopause. 2011;18:638-45.

18. Ko YL, Lin SC, Lin PC. Effect of auricular acupressure for postpartum insomnia: an uncontrolled clinical trial. J Clin Nurs. 2016;25:332-9. 
19. He W, Wang X, Shi H, Shang H, Li L, Jing X, Zhu B. Auricular acupuncture and vagal regulation. Evid Based Complement Alternat Med. 2012;2012. https://doi.org/10.1155/2012/786839.

20. Wei CY, Chung TC, Wu SC, Chung CF, Wu WP. The subjective sleep quality and heart rate variability in hemodialysis patients. Ren Fail. 2011;33:109-17.

21. Wu Y, Zou C, Liu X, Wu X, Lin Q. Auricular acupressure helps improve sleep quality for severe insomnia in maintenance hemodialysis patients: a pilot study. J Altern Complement Med. 2014;20:356-63.

22. Zou C, Yang L, Wu Y, Su G, Chen S, Guo X, Wu X, Liu X, Lin Q. Auricular acupressure on specific points for hemodialysis patients with insomnia: a pilot randomized controlled trial. PLoS One. 2015;10:e0122724. https://doi. org/10.1371/journal.pone.0122724.

23. Singh S, Choi P, Power A, Ashby D, Cairns T, Griffith M, McLean A, Palmer A, Taube D, Duncan N. Ten-year patient survival on maintenance haemodialysis: association with treatment time and dialysis dose. J Nephrol. 2013;26:763-70.

24. Lukowsky LR, Kheifets L, Arah OA, Nissenson AR, Kalantar-Zadeh K. Patterns and predictors of early mortality in incident hemodialysis patients: new insights. Am J Nephrol. 2012;35:548-58.

25. American Psychiatric Association. Diagnostic and Statistical Manual of Mental Disorders: DSM-5. 5th ed. Arlington: American Psychiatric Association; 2013.

26. McCarney R, Warner J, lliffe S, van Haselen R, Griffin M, Fisher P. The Hawthorne Effect: a randomised, controlled trial. BMC Med Res Methodol. 2007:7:30.

27. Mitchell TR, Daniels D. Motivation. In: Borman WC, Ilgen DR, Klimoski RJ, editors. Handbook of psychology. Hoboken: Wiley; 2003. p. 229.

28. Yang G, Lin S, Wu Y, Zhang S, Wu X, Liu X, Zou C, Lin Q. Auricular acupressure helps alleviate xerostomia in maintenance hemodialysis patients: a pilot study. J Altern Complement Med. 2017;23:278-84.

29. Cao H, Pan X, Li H, Liu J. Acupuncture for treatment of insomnia: a systematic review of randomized controlled trials. J Altern Complement Med. 2009;15:1171-86.

30. Gao XY, Wang L, Gaischek I, Michenthaler Y, Zhu B, Litscher G. Brainmodulated effects of auricular acupressure on the regulation of autonomic function in healthy volunteers. Evid Based Complement Alternat Med. 2012; 2011. https://doi.org/10.1155/2012/714391.

31. Wang L, Cheng W, Sun Z, Xu Y, Cheng G, Gaischek I, Kuang H, Litscher G. Ear acupressure, heart rate, and heart rate variability in patients with insomnia. Evid Based Complement Alternat Med. 2013;2013. https://doi.org/ $10.1155 / 2013 / 763631$.

32. He W, Jing XH, Zhu B, Zhu XL, Li L, Bai WZ, Ben H. The auriculo-vagal afferent pathway and its role in seizure suppression in rats. BMC Neurosci. 2013;14:85.

33. Zhou LQ, Zhao BX. Nomenclature and location of auricular points. Beijing, China: Standard Publishing House; 2008.

34. Wang JL. Clinical epidemiology: design, measurement and evaluation in clinical research. 3rd ed. Shanghai: Shanghai Science and Technology Press; 2009. p. 156-65.

35. Buysse DJ, Reynolds CF, Monk TH, Berman SR, Kupfer DJ. The Pittsburgh Sleep Quality Index: a new instrument for psychiatric practice and research. Psychiatry Res. 1989;28:193-213.

36. Liu XC, Tang MQ, Hu L. Reliability and validity of the Pittsburgh Sleep Quality Index [Chinese]. Chinese Journal of Psychiatry. 1996;2:103-7.

37. Tsai PS, Wang SY, Wang MY, Su CT, Yang TT, Huang CJ, Fang SC. Psychometric evaluation of the Chinese version of the Pittsburgh Sleep Quality Index (CPSQI) in primary insomnia and control subjects. Qual Life Res. 2005;14:1943-52.

38. Buysse DJ, Germain A, Moul DE, Franzen PL, Brar LK, Fletcher ME, Begley A, Houck PR, Mazumdar S, Reynolds CF 3rd, Monk TH. Efficacy of brief behavioral treatment for chronic insomnia in older adults. Arch Intern Med. 2011;171:887-95.

39. Chen XM. Management of hemodialysis patients. In: Blood purification standard operating procedure (SOP). Beijing: Ministry of Health of the People's Republic of China; 2010. p. 70-2.

40. Standardization Administration of the People's Republic of China. The name and location of auricular acupoints (GB/T13734-2008). Beijing: State Bureau of Technical Supervision; 2008.

41. Standardization Administration of the People's Republic of China. Standardized manipulation of acupuncture and moxibustion. Part 3: auricular acupuncture (GB/T21709.3-2008). Beijing: State Bureau of Technical Supervision; 2008.
42. Buysse DJ, Ancoli-Israel S, Edinger JD, Lichstein KL, Morin CM. Recommendations for a standard research assessment of insomnia. Sleep. 2006;29:1155-73.

43. Wang SY, Zang XY, Liu JD, Cheng M, Shi YX, Zhao Y. Indicators and correlates of psychological disturbance in Chinese patients receiving maintenance hemodialysis: a cross-sectional study. Int Urol Nephrol. 2015;47:679-89.

\section{Submit your next manuscript to BioMed Central and we will help you at every step:}

- We accept pre-submission inquiries

- Our selector tool helps you to find the most relevant journal

- We provide round the clock customer support

- Convenient online submission

- Thorough peer review

- Inclusion in PubMed and all major indexing services

- Maximum visibility for your research

Submit your manuscript at www.biomedcentral.com/submit
Biomed Central 